'Departamento de Dermatología, Facultad de Medicina,

Universidad de Chile. Santiago, Chile.

${ }^{2}$ Servicio de Dermatología Hospital Clínico San Borja Arriarán. Santiago, Chile.

${ }^{3}$ Departamento de Anatomía Patológica, Facultad de Medicina, Universidad de Chile. Santiago, Chile.

${ }^{4}$ Servicio de Anatomía Patológica, Hospital Clínico San Borja Arriarán. Santiago, Chile.

Trabajo no recibió financiamiento. Los autores declaran no tener conflictos de interés.

Recibido el 7 de abril de 2020, aceptado el 31 de agosto de 2020.

Correspondencia a: Javier Arellano javierarlo@uchile.cl

\section{Lupus eritematoso asociado a eritema multiforme: Síndrome de Rowell. Reporte del primer caso en Chile}

\author{
LORETO HEREDIA ${ }^{1}$, JAVIER ARELLANO ${ }^{2}$, YAMILE CORREDOIRA ${ }^{3,4}$
}

\section{Lupus erythematosus associated with erythema multiforme or Rowell's syndrome. Report of one case}

Rowell's syndrome is characterized by an association of lupus erythematosus and erythema multiforme, with distinctive laboratory findings. Its treatment is similar to lupus. We report a 16-year-old female presenting with skin lesions, laboratory and histology consistent with this entity. Because of the spread of cutaneous involvement and development of epidermal detachment, she required therapy with intravenous corticosteroids and gamma globulin.

(Rev Med Chile 2020; 148: 1690-1693)

Key words: Erythema Multiforme; Lupus Erythematosus, Cutaneous; Lupus Erythematosus, Systemic.
L a asociación entre lupus eritematoso (LE) y eritema multiforme (EM) fue definida por primera vez en 1963 por Rowell, quien describió el síndrome a partir de cuatro pacientes que se presentaron con lesiones tipo EM asociadas a LE discoide ${ }^{1}$. A la fecha, sigue siendo una entidad muy poco frecuente, con criterios diagnósticos que se han ido estableciendo en base a los reportes de casos que han sido publicados en la literatura. El objetivo de este trabajo es presentar un caso de síndrome de Rowell (SR), siendo, a nuestro entender, el primer caso reportado en Chile, y comentar la presentación clínica, los criterios diagnósticos actuales y el manejo terapéutico.

Se contó con consentimiento informado por escrito de la paciente y sus padres para su publicación.

\section{Caso clínico}

Paciente de 16 años, de sexo femenino, sin cuadros de herpes o infecciones recientes, ni historia de uso de fármacos. Consultó por un cuadro de tres días de evolución de compromiso del estado general, fiebre y lesiones cutáneas. Al examen físico, presentaba en cara un eritema de predominio malar y de dorso nasal cubierto por costras mielicéricas. En el borde bermellón de los labios eritema y descamación fina, sin compromiso de mucosa bucal, lingual, ni de otras mucosas. En tronco y extremidades máculas eritematosas targetoides atípicas, con centro violáceo, confluentes, de distribución simétrica. Signo de Nikolsky negativo. En las falanges distales de ambas manos destacaba la presencia de placas infiltradas eritematovioláceas (Figura 1).

Dentro de los exámenes de laboratorio destacaba un ANA positivo 1/160 patrón moteado, anticuerpos anti Ro positivos, complemento C3 y $\mathrm{C} 4$ bajos, $\mathrm{y}$ factor reumatoide en $530 \mathrm{UI} / \mathrm{mL}$ (valor normal 10-30). El anti-DNA por inmunofluorescencia resultó negativo. Sin hallazgos en la historia, examen físico ni en laboratorio que revelara compromiso de otros órganos. Se realizó biopsia de las lesiones targetoides, cuya histología mostró debajo y sobre la epidermis una banda eosinófila de queratinocitos superficiales necróticos, y en 
la unión dermo-epidérmica, daño vacuolar de interfase con infiltrado linfocitario perivascular y queratinocitos necróticos, hallazgos compatibles con eritema multiforme menor (Figura 2). La inmunofluorescencia directa (IFD) resultó nega- tiva. La biopsia de las lesiones en las falanges fue compatible con eritema pernio.

El cuadro clínico y los hallazgos descritos son compatibles con un SR, que se caracteriza por la asociación de LE con lesiones tipo EM.

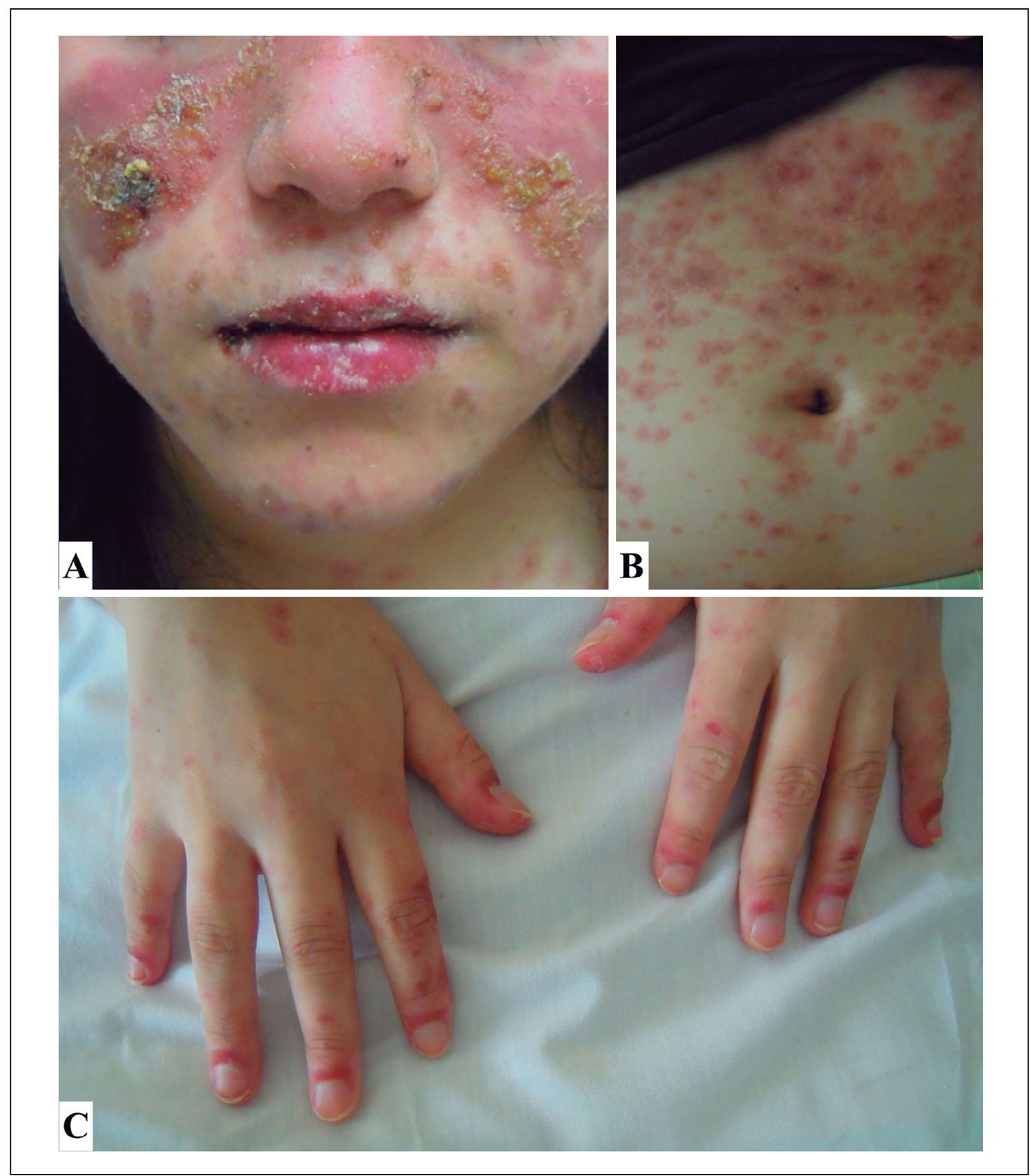

Figura 1. A: Eritema costroso de predominio malar y de dorso nasal. B: Máculas targetoides confluentes en tronco. C: Eritema pernio en falanges distales. 


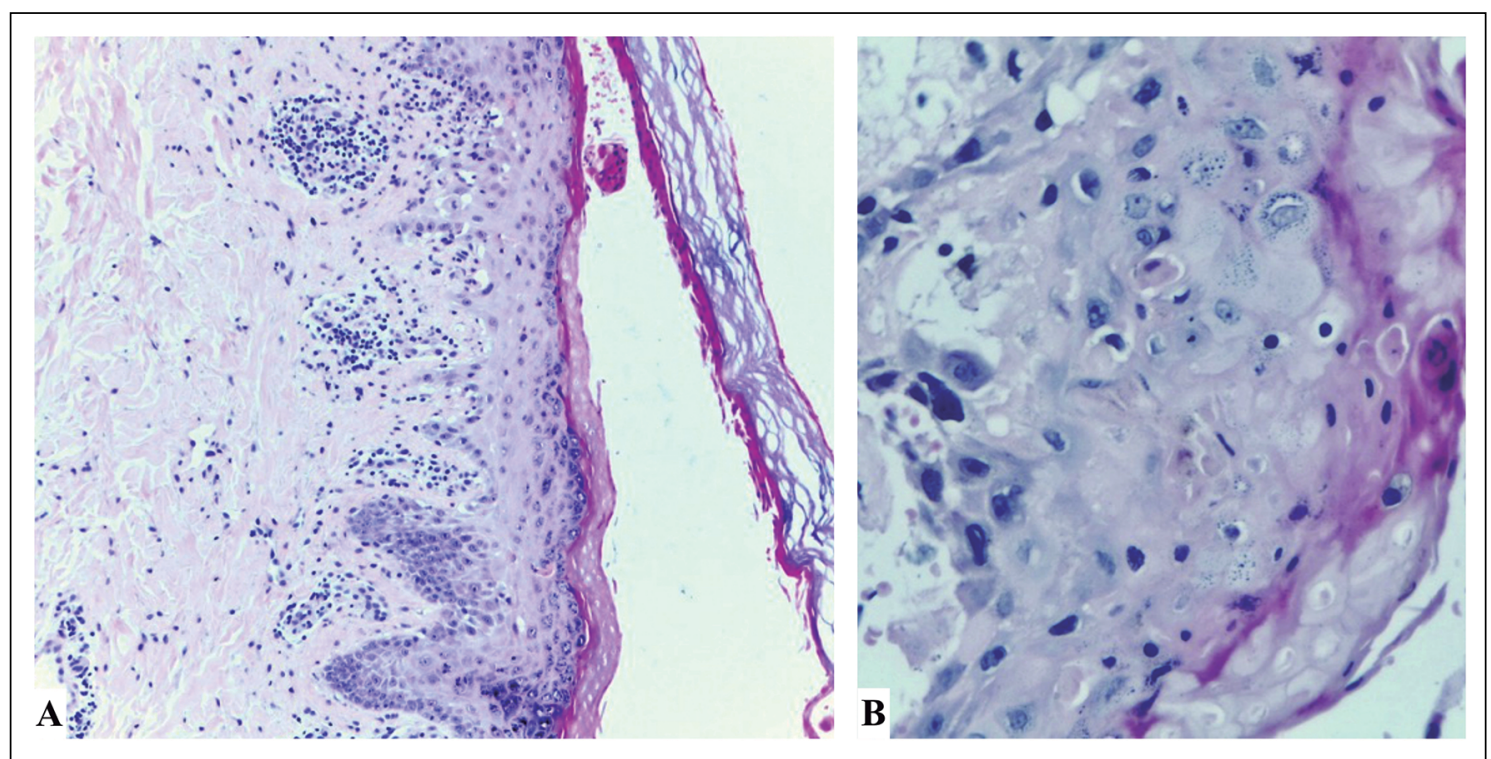

Figura 2. Histología. A: Banda eosinófila de queratinocitos necróticos bajo y sobre la epidermis, en unión dermoepidérmica daño vacuolar de interfase con infiltrado linfocitario perivascular y queratinocitos necróticos. B: Daño vacuolar de interfase y queratinocitos necróticos.

La paciente se hospitalizó para terapia y observación y se inició tratamiento con prednisona $0,5 \mathrm{mg} / \mathrm{kg} /$ día. Al inicio presentó una evolución tórpida, con lesiones que progresaron comprometiendo hasta $70 \%$ de la superficie corporal y con desprendimiento epidérmico asociado. Destacó que las mucosas se mantuvieron sanas durante toda la evolución del cuadro y que no hubo otros fármacos involucrados. Se consideró necesario el tratamiento con corticoides endovenosos y gammaglobulina en dosis de $1 \mathrm{~g}$ por kilo al día durante tres días, evolucionando de manera favorable con reepitelización, resolución de las lesiones cutáneas y sin presentar complicaciones infecciosas ni secuelas.

\section{Discusión}

El SR es una entidad que se caracteriza por la asociación entre LE y EM, en presencia de marcadores inmunológicos específicos ${ }^{2}$. La manifestaciones cutáneas más habitualmente descritas en la literatura son lesiones targetoides tipo eritema multiforme y eritema pernio ${ }^{3}$. El compromiso cutáneo puede evolucionar a desprendimiento epidérmico, como en el caso presentado, sin cumplir criterios de reacción adversa a fármacos ${ }^{4}$. Dentro de los hallazgos de laboratorio, lo más frecuente es el ANA patrón moteado. A la histología, las lesiones targetoides son compatibles con un EM, pudiendo observarse queratinocitos necróticos, ampollas subepidérmicas y un infiltrado linfocitico escaso ${ }^{3}$.

En cuanto a la epidemiología es similar al LE, siendo la incidencia mayor en mujeres que en hombres, en relación 8:1 y la edad de presentación promedio es entre 30 y 40 años. Existen alrededor de 90 casos reportados en la literatura ${ }^{5}$. Los criterios diagnósticos más recientes son los de Torchia et al. descritos en 2012 (Tabla 1). Se requiere la presencia de los 4 criterios mayores y al menos uno menor para el diagnóstico ${ }^{5}$. La paciente presentada cumplía con todos estos criterios diagnósticos al momento del ingreso.

La respuesta terapéutica y el pronóstico de esta entidad se han descrito como similares a las de las distintas variantes del LE, es decir, una respuesta al tratamiento variable y con recurrencias frecuentes ${ }^{6}$. El manejo está enfocado en el LE, con corticoides sistémicos y antimaláricos, asociados a otros inmunosupresores. En aquellos pacientes en que las lesiones progresan a desprendimiento epidérmico, como en el caso descrito, se ha re- 
Tabla 1. Criterios diagnósticos de síndrome de Rowell (Torchia et al.)

\begin{tabular}{|ll|}
\hline Criterios mayores & Criterios menores \\
$\begin{array}{l}\text { Presencia de lupus cutáneo crónico (lupus discoide o eritema } \\
\text { pernio) }\end{array}$ & Ausencia de infecciones o fármacos desencadenantes \\
$\begin{array}{l}\text { Presencia de lesiones tipo EM (lesiones en diana típicas O O } \\
\text { atípicas) }\end{array}$ & $\begin{array}{l}\text { Ausencia de lesiones típicas de eritema multiforme (acral y } \\
\text { mucosas) }\end{array}$ \\
$\begin{array}{l}\text { Positividad para al menos un anticuerpo: ANA con patrón } \\
\text { moteado, anti- Ro o anti-La }\end{array}$ & $\begin{array}{l}\text { Presencia de, por lo menos, un criterio LE sistémico de la } \\
\text { Academia Americana de Reumatología (además del eritema } \\
\text { discoide y ANA positivo), con exclusión de fotosensibilidad, } \\
\text { erupción malar y úlceras orales }\end{array}$ \\
\hline IFD negativa en lesiones de tipo EM & \\
\hline
\end{tabular}

LE: lupus eritematoso; EM: eritema multiforme; IFD: inmunofluorescencia directa.

portado el tratamiento con gamaglobulina con buena respuesta ${ }^{3}$.

Respecto al diagnóstico diferencial de este caso clínico, se deben considerar las distintas etiologías de erupciones bulosas graves en pacientes con lupus ${ }^{4}$. Entre ellos, el lupus eritematoso buloso, en este, las vesículas se producen en áreas fotoexpuestas y la IFD es positiva. En la necrolisis epidérmica tóxica (NET) hay compromiso grave de más de una mucosa y, en la mayoría de los casos, se detecta un fármaco asociado. En el lupus NET-Like hay compromiso de mucosa no queratinizada y la IFD es característicamente positiva. El SR tiene hallazgos muy característicos: Eritema pernio, ANA positivo patrón moteado, anti Ro o anti La positivos, factor reumatoide positivo e IFD negativa.

Existen autores que han puesto en duda la existencia de este síndrome, planteando que algunos casos publicados después de la descripción original de Rowell corresponderían a un subtipo de lupus eritematoso cutáneo subagudo con lesiones targetoides, más que a una entidad distintiva, y que los casos restante podrían ser una coincidencia entre EM y lupus eritematoso que no justifica la clasificación como un síndrome independiente ${ }^{7}$. El objetivo de los últimos criterios publicados es justamente excluir a esos casos que se desvían de la descripción original. Consideramos de interés publicar este caso que cumple con todos los criterios de Torchia et al, de manera de documentar su presentación y ampliar los antecedentes para la discusión.

Creemos importante tener en consideración esta entidad dentro de los diagnósticos diferencia- les ante lesiones targetoides y erupciones bulosas en pacientes con antecedentes de lupus o con clínica compatible, lo que facilitará el diagnóstico precoz y tratamiento dirigido.

\section{Referencias}

1. Rowell N, Anderson J. Lupus Erythematosus and Erythema Multiforme-like Lesions. Arch Dermatol 1963; 88 (2): 176-80.

2. Kim S, Magro C, Granstein R, Bass A, Erkan D. Systemic lupus erythematosus associated with Rowell's syndrome. HSS J 2013; 9 (3): 289-92.

3. Aydogan K, Karadogan S, Balaban S, Tunali S. Lupus erythematosus associated with erythema multiforme: report of two cases and review of the literature. J Eur Acad Dermatol Venereol 2005; 19 (5): 621-7.

4. Romero L, Bari O, Forbess Smith C, Schneider J, Cohen $\mathrm{P}$. Toxic epidermal necrolysis-like acute cutaneous lupus erythematosus: report of a case and review of the literature. Dermatol Online J 2018; 15; 24 (5). Disponible en: https://escholarship.org/ [Consultado el 5 de noviembre de 2019].

5. Torchia D, Romanelli P, Kerdel F. Erythema multiforme and Stevens-Johnson syndrome/toxic epidermal necrolysis associated with lupus erythematosus. J Am Acad Dermatol 2012; 67 (3): 417-21.

6. Bhat R, Varma C, Bhatt S, Balachandran C. Rowell syndrome. Indian Dermatol Online J 2014; 5 (1): 33-5. Disponible en www.idoj.in [Consultado el 5 de noviembre de 2019].

7. Antiga E, Caproni M, Bonciani D, Bonciolini V, Fabbri $\mathrm{P}$. The last word on the so-called "Rowell"s syndrome'? Lupus 2011; 21 (6): 577-85 [Consultado el 4 de junio de 2020]. 